PROCEEDINGS OF THE

AMERICAN MATHEMATICAL SOCIETY

Volume 132, Number 9, Pages 2517-2527

S 0002-9939(04)07302-2

Article electronically published on April 8, 2004

\title{
A CUSP SINGULARITY WITH NO GALOIS COVER BY A COMPLETE INTERSECTION
}

\author{
DAVID E. ANDERSON
}

(Communicated by Ronald A. Fintushel)

\begin{abstract}
With an explicit example, we confirm a conjecture by Neumann and Wahl that there exist cusps with no Galois cover by a complete intersection. Some computational techniques are reviewed, and a method for deciding whether a given cusp has a complete intersection Galois cover is developed.
\end{abstract}

\section{INTRODUCTION}

In [7, Neumann and Wahl prove that the universal abelian cover of every quotient-cusp is a complete intersection, and conjecture generally that a similar result holds for any $\mathbb{Q}$-Gorenstein normal surface singularity whose link is a rational homology sphere. (For discussions of these singularities and others known to have complete intersection abelian covers, see [5], [6], and [7]. By a cover of a complex surface singularity we mean a finite cover of a germ of the singularity, branched only at the singular point.) Cusps are not included in the scope of this conjecture, since their links are not $\mathbb{Q}$-homology spheres. So one might ask whether similar results hold in their case. In the same article (Proposition 4.1), it is proved that every cusp has a cover by a complete intersection, but there exist cusps with no abelian cover by a complete intersection. The authors go on to conjecture that some cusps do not even have a Galois cover by a complete intersection.

In the present paper, we exhibit a cusp confirming this latter conjecture: We show that the cusp classified by the cycle $(8,2,4,3,12)$ provides the desired example. The existence of such a singularity precludes a natural generalization of the main theorem of [7] from the case of quotient-cusps to a statement about cusps. More generally, Conjecture 1.1 of [7] does not naturally generalize to the case of singularities whose links are not rational homology spheres.

We begin with a brief review of singularity links and cusps; for more details, see [4] and [7.

Consider a germ of a normal complex suface singularity $(V, p)$. A good resolution of the singularity is a map $\pi: \bar{V} \rightarrow V$ such that $\bar{V}$ is nonsingular, $\pi: \pi^{-1}(V-\{p\}) \rightarrow$ $V-\{p\}$ is an isomorphism, and $\pi^{-1}(p)$ is a complex curve whose only singularities are normal crossings and all of whose components (the exceptional curves) are nonsingular. The good resolution with fewest exceptional curves is unique and is

Received by the editors December 6, 2001 and, in revised form, January 8, 2003.

2000 Mathematics Subject Classification. Primary 14B05, 14J17.

Supported by the NSF's VIGRE Fellowship through the Columbia University Department of Mathematics. The author is greatly indebted to Professor Walter Neumann for his guidance. 
called the minimal good resolution. The resolution graph is the weighted graph whose vertices stand for the exceptional curves weighted according to their selfintersection numbers, and whose edges correspond to intersections of exceptional curves. A link of the singularity $(V, p)$ is the oriented 3-manifold $M$ that forms the boundary of a closed regular neighborhood of $p$. Using the plumbing procedure described in [4], the singularity link $M$ can be reconstructed from the resolution graph. The topology of the minimal good resolution $\bar{V}$ is determined by the oriented manifold $M$ (see [4]).

The cusp singularities are those whose resolution graphs are cyclic and all of whose exceptional curves are rational. Equivalently, the link of a cusp is a torus bundle over the circle, with monodromy $A$ having trace $\geq 3$. A cusp singularity also has a dual cusp whose link is $-M$ ( $M$ with reversed orientation), a torus bundle with monodromy $A^{-1}$. (In this we follow the orientation convention of [4].)

The plumbing calculus of [4] reconstructs the monodromy $A$ of a cusp's link as follows: Up to conjugation, the classifying matrix is

$$
A=\left(\begin{array}{cc}
-e_{k} & 1 \\
-1 & 0
\end{array}\right) \ldots\left(\begin{array}{cc}
-e_{1} & 1 \\
-1 & 0
\end{array}\right)
$$

where $e_{1}, \ldots, e_{k}$ are the weights of the vertices of the resolution cycle. We shall call $\left(-e_{1}, \ldots,-e_{k}\right)$ the resolution cycle of the link determined by $A$, or more concisely, the cycle of $A$.

Finally, a germ of a complex surface is a complete intersection (abbreviated "CI") if there exists an imbedding $(V, p) \hookrightarrow\left(\mathbb{C}^{n}, 0\right)$ such that $V$ is given by $n-2$ equations. It follows from Karras [2] that a cusp is a CI if and only if the resolution cycle of the dual cusp has length $\leq 4$ (see [7]).

A useful tool in examining Galois covers of a cusp is the fact, Proposition 2.3 that if a cusp is a Galois cover of a given cusp, then the cusp dual to the covering cusp is also a Galois cover. Thus a cusp has no Galois cover by a CI if and only if it has no Galois cover by a cusp with cycle of length at most 4 .

The topology of the minimal good resolution of a cusp singularity is determined (up to orientation reversal, i.e., replacing the cusp by its dual) by the fundamental group of the singularity link $M$ [1], 44. So the topological question of whether no CI Galois cover exists reduces to an algebraic problem. The fundamental group $\pi_{1}(M)$ is isomorphic to the semidirect product $\mathbb{Z}^{2} \rtimes_{A} \mathbb{Z}$; Galois coverings correspond to normal subgroups of $\pi_{1}(M)$. The question now becomes, "Is there a normal subgroup of $\mathbb{Z}^{2} \rtimes_{A} \mathbb{Z}$ whose classifying matrix is conjugate to $B=\left(\begin{array}{cc}-e_{k} & 1 \\ -1 & 0\end{array}\right) \ldots\left(\begin{array}{cc}-e_{1} & 1 \\ -1 & 0\end{array}\right)$, for some $e_{1}, \ldots, e_{k}$ with $k \leq 4$ and each $e_{i} \leq-1$ ?"

To find and verify an example that answers this question negatively, we proceed as follows. In Section 2, we state and prove some algebraic facts about normal subgroups of $\pi_{1}(M)$, and arrive at a condition on the factorization of the trace of the monodromy that reduces the number of candidates for normal subgroups. In Section 3] we discuss the algorithms developed in [4] for calculating the plumbing cycle of a cusp given the monodromy of its link, and for determining the cycle of the dual cusp given the cycle of the original. Guided by conditions on the trace, we then posit a matrix to serve as a candidate for the monodromy of the link of a cusp with no Galois cover by a CI. Finally, in Section 4 we systematically examine the normal subgroups of the fundamental group of this link; that is, we compute the monodromy matrix for each subgroup and verify that its cycle is longer than 4 . 


\section{Algebraic preliminaries}

The fundamental group of the torus bundle $M$ with monodromy $A$ is $\mathbb{Z}^{2} \rtimes_{A} \mathbb{Z}$, where the group operation in the semidirect product is given by $(x, n)(y, m):=$ $\left(x+A^{n} y, n+m\right)$. Throughout this paper, we shall denote the monodromy matrix of $M$ by $A$, and the group $\pi_{1}(M)$ by $G$. The subgroup $\mathbb{Z}^{2} \subset G$ is a characteristic subgroup of $G$, since it is the kernel of the abelianization of $G$ modulo torsion.

In discussing subgroups of $\pi_{1}(M)$, we follow the terminology of [7]: a proper subgroup of $\mathbb{Z}^{2} \rtimes \mathbb{Z}$ that surjects onto $\mathbb{Z}$ is called a covering in the fiber (or a fiberwise cover), and a subgroup that contains $\mathbb{Z}^{2}$ is a covering in the base. Also, an isomorphism of covers will mean an isomorphism of the corresponding subgroups as groups, not necessarily by an isomorphism induced by an automorphism of $G$.

Proposition 2.1. Every covering is

(a) a (Galois) covering in the base followed by a covering in the fiber, and

(b) up to isomorphism, a covering in the fiber followed by a covering in the base.

Proof. (a) Let $G$ be the fundamental group of $M$, and let $N$ be the subgroup corresponding to the covering (i.e., the fundamental group of the cover), let $L=$ $N \cap \mathbb{Z}^{2}$, and let $n=[\mathbb{Z}: \pi(N)]$, where $\pi$ is the projection of $G$ onto $\mathbb{Z}$. We can construct the following diagram of short exact sequences:

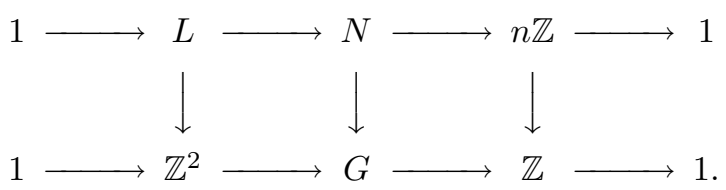

We want an $\tilde{N}$ to satisfy $N \subset \tilde{N} \subset G$, with a fiber factor equal to that of $G$ and base factor equal to that of $N$ :

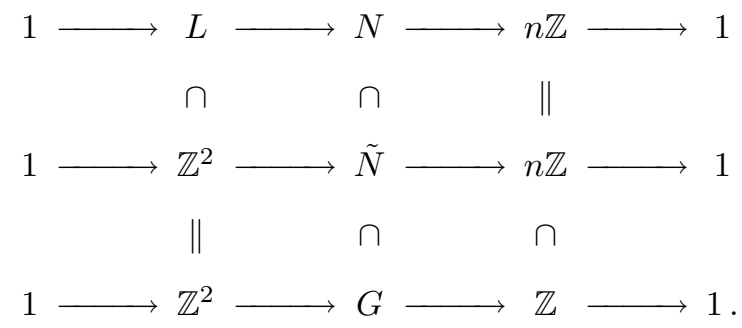

The diagram is satisfied by taking $\tilde{N}$ to be the kernel of the natural homomorphism from $G$ to $\mathbb{Z} / n$ given by $(x, y) \mapsto y(\bmod n)$. Note that $\tilde{N}$ is normal in $G$; thus if $N$ is normal in $G$ (i.e., the original cover is Galois), then $N$ is normal in $\tilde{N}$ and the fiberwise cover is also Galois.

(b) Now we want $\tilde{N}^{\prime}$ to satisfy

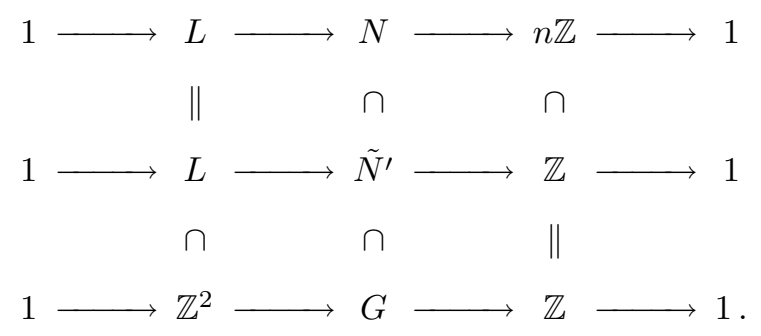


$N$ is generated by $L$ and $(x, n)$ for some $x \in \mathbb{Z}^{2}$. Any subgroup $\tilde{N}^{\prime}$ that fits in the above horizontal exact sequence will be generated by $L$ and $\left(x^{\prime}, 1\right)$ for some $x^{\prime} \in \mathbb{Z}^{2}$. This $\tilde{N}^{\prime}$ will contain $N$ only if $(x, n)$ is in the group generated by $L$ and $\left(x^{\prime}, 1\right)^{n}$, and an $x^{\prime}$ with this property cannot always be found. However, $N$ is isomorphic to the subgroup of $\mathbb{Z}^{2} \rtimes_{A} \mathbb{Z}$ generated by $L$ and $(0, n)$, and if we replace $N$ by this subgroup, then we may take $\tilde{N}^{\prime}$ as the subgroup generated by $L$ and $(0,1)$.

Using Proposition 2.1(b), we can restrict our search to covers of degree at most four in the base: If $\tilde{N}^{\prime}$ has monodromy $B$, then $N=L \rtimes_{B} n \mathbb{Z}=L \rtimes_{B^{n}} \mathbb{Z}$ has monodromy $B^{n}$, which has cycle of length $n$ times that of $B$. Covers of degree greater than four thus have cycles of length at least 5 . (This is easy to see, since a covering of degree $n$ in the base just makes the cycle repeat $n$ times.)

Proposition 2.1. (a) tells us that we need only look at normal covers in the fiber; this condition on $N$, together with the following, will also limit our search.

Proposition 2.2. Let $N$ be a subgroup of $G$, with $L$ and $n$ as above. Then $N$ is normal if and only if $A L=L$ and $\left(A^{n}-I\right) \mathbb{Z}^{2} \subset L \subset \mathbb{Z}^{2}$.

Proof. $N$ is generated by $L$ and $(x, n)$ for some $x$, and $L$ is a characteristic subgroup of $N$; so $N$ is normal only if $L$ is normal. $L \rightarrow(L, 0)$, and so conjugation by $(0,1)$ gives

$$
(0,1)^{-1}(L, 0)(0,1)=\left(A^{-1} L, 0\right) .
$$

$L$ is therefore preserved by conjugation if and only if it is preserved by $A$.

Next, conjugation of $(x, n)$ by $(b, 0)$ for any $b \in \mathbb{Z}^{2}$ gives

$$
(b, 0)^{-1}(x, n)(b, 0)=\left(-b+x+A^{n} b, n\right) .
$$

Therefore, we must have

$$
\left(A^{n}-I\right) \mathbb{Z}^{2} \subset L
$$

The converse is easily verified.

The above propositions let us give the proof of the following:

Proposition 2.3. If a cusp is a Galois cover of a given cusp, then its dual cusp is also such a Galois cover.

Proof. Given a cusp with monodromy $A$, a covering in the fiber with fiber group $L=(A-I) \mathbb{Z}^{2}$ is called a discriminant cover (see [7]). Let us call a cover of $M$ a sub-discriminant cover if it lies between $M$ and a discriminant cover (i.e., it is covered by a discriminant cover). Then Propositions 2.1 and 2.2 imply that any Galois cover is the result of a cover in the base followed by a sub-discriminant cover in the fiber, and that any such cover is a Galois cover. In Section 4 of [7] it is shown that the collection of sub-discriminant covers of a given cusp is closed under taking duals; so the proposition follows.

Assuming $n \leq 4$ in our desired example, Proposition 2.2 now says that we must consider subgroups of $\mathbb{Z}^{2} /\left(A^{n}-I\right) \mathbb{Z}^{2}$ for each $n$. To limit the number of subgroups $L$ satisfying the given conditions, then, we would like to keep $\mathbb{Z}^{2} /\left(A^{n}-I\right) \mathbb{Z}^{2}$ as simple as possible. It is easy to see that

Proposition 2.4. $\left|\mathbb{Z}^{2} /\left(A^{n}-I\right) \mathbb{Z}^{2}\right|=\left|2-P_{n}(\operatorname{tr} A)\right|$, where $P_{n}(\operatorname{tr} A)$ is a polynomial in the trace of $A$. 
In particular, we have for $n=1,2,3,4$,

$$
\begin{aligned}
& {\left[\mathbb{Z}^{2}:\left(A^{1}-I\right) \mathbb{Z}^{2}\right]=x-2,} \\
& {\left[\mathbb{Z}^{2}:\left(A^{2}-I\right) \mathbb{Z}^{2}\right]=(x-2)(x+2),} \\
& {\left[\mathbb{Z}^{2}:\left(A^{3}-I\right) \mathbb{Z}^{2}\right]=(x-2)(x+1)^{2},} \\
& {\left[\mathbb{Z}^{2}:\left(A^{4}-I\right) \mathbb{Z}^{2}\right]=x^{2}(x-2)(x+2),}
\end{aligned}
$$

where $x$ is the trace of $A$. By Proposition 2.2 these are the maximal indices (in the fiber) of normal subgroups. To minimize the number of potentially normal subgroups, we minimize the number of factors of $\left|\mathbb{Z}^{2} /\left(A^{n}-I\right) \mathbb{Z}^{2}\right|$; let us require that $x$ and $x-2$ be prime, $x+2$ be three times a prime, and $x+1$ be twice a prime. For convenience, let us denote the prime factors of $\left|\mathbb{Z}^{2} /\left(A^{n}-I\right) \mathbb{Z}^{2}\right|$ as follows:

$$
\begin{aligned}
p & =\operatorname{tr} A, \\
q & =p-2, \\
3 r & =p+2, \\
2 s & =p+1,
\end{aligned}
$$

where $p, q, r$, and $s$ are all prime.

\section{CyCles}

We now turn our attention to the cycle of a cover. Recall that the resolution graph for the link of a cusp singularity is cyclic, and each vertex with weight $e_{i}$ corresponds to a joining of trivial torus bundles by matrices of the form $\left(\begin{array}{cc}-e_{i} & 1 \\ -1 & 0\end{array}\right)$. The monodromy of the entire link, then, is

$$
B=\left(\begin{array}{cc}
b_{k} & 1 \\
-1 & 0
\end{array}\right) \cdots\left(\begin{array}{cc}
b_{1} & 1 \\
-1 & 0
\end{array}\right),
$$

where $b_{i}=-e_{i}$. Written this way, $B$ classifies the link of a complete intersection (up to orientation) iff $k \leq 4$. Now we need a method for expanding a given matrix in this form and an algorithm for finding the corresponding dual matrix. The tools for both are described in 44; we summarize briefly here.

Given $B=\left(\begin{array}{ll}a & b \\ c & d\end{array}\right)$ with trace $t$, let

$$
\omega=\frac{a-d+\sqrt{t^{2}-4}}{2 b} .
$$

To find the cycle, first compute the "continued fraction with minus signs" expansion of $\omega$ :

$$
\omega=a_{0}-\frac{1}{a_{1}-\frac{1}{a_{2}-\frac{1}{\ddots}}},
$$

and let $\left[c_{1}, \ldots, c_{l}\right]$ be the shortest period of this ultimately periodic continued fraction. Put $C=\left(\begin{array}{cc}c_{l} & 1 \\ -1 & 0\end{array}\right) \ldots\left(\begin{array}{cc}c_{1} & 1 \\ -1 & 0\end{array}\right)$, with trace $t^{\prime}$, and solve

$$
\frac{t+\sqrt{t^{2}-4}}{2}=\left(\frac{t^{\prime}+\sqrt{\left(t^{\prime}\right)^{2}-4}}{2}\right)^{n}
$$

for $n$. Then the cycle of $B$ is given by concatenating $\left(c_{1}, \ldots, c_{l}\right)$ with itself $n$ times.

Since this method depends on finding the period of a continued fraction, computation will be greatly simplified if the continued fraction is purely periodic. The 
following proposition gives a condition for pure periodicity of a continued fraction with minus signs. The proof is analogous to that of a similar statement about ordinary continued fractions; see, for example, Theorem 11.5.2 in [3].

Proposition 3.1. The continued fraction (with minus signs) expansion of a quadratic irrationality $x=(P+\sqrt{n}) / Q$ is purely periodic if and only if $x>1$ and $0<\bar{x}<1$, where $\bar{x}$ is the conjugate of $x$ in $\mathbb{Q}[\sqrt{n}]$.

In relation to the cycle of $B$, Proposition 3.1 requires

$$
\frac{(a-d)+\sqrt{t^{2}-4}}{2 b}>1 \text { and } \frac{2 b}{(a-d)-\sqrt{t^{2}-4}}>1,
$$

where $t=a+d$. For $t \geq 3$ and $a, b, d \in \mathbb{Z}$, this reduces to the condition

$$
a>b>-d \geq 0 .
$$

Since the dual to a Galois cover of a given cusp is also a Galois cover, we must inspect the dual cycle as well. If $\left(b_{1}, \ldots, b_{k}\right)$ is the cycle of $B$, then from [1] we know $b_{i} \geq 2$ for each $i$, and $b_{i} \geq 3$ for some $i$. Write the cycle as

$$
\left(b_{1}, \ldots, b_{k}\right)=(m_{1}+3, \underbrace{2, \ldots, 2}_{n_{1}}, m_{2}+3, \ldots, m_{s}+3, \underbrace{2, \ldots, 2}_{n_{s}}) ;
$$

the dual cycle is then given by

$$
\left(d_{1}, \ldots, d_{l}\right)=(n_{s}+3, \underbrace{2, \ldots, 2}_{m_{s}}, n_{s-1}+3, \ldots, n_{1}+3, \underbrace{2, \ldots, 2}_{m_{1}}) .
$$

As is well known, the length of the dual cycle can be written simply in terms of the entries of the original cycle as $\sum_{i=1}^{n}\left(b_{i}-2\right)$.

For our example, we want to find a matrix whose trace satisfies the primality condition (2), and whose entries satisfy the condition for immediate periodicity given in (6).

First we choose a trace. The first three numbers satisfying (2) are 13, 1621, and 6661. Computation time increases rapidly with the size of the trace, but 13 is too small to yield a long enough cycle; so we use 1621.

Now we construct a matrix in $S L_{2}(\mathbb{Z})$ with trace 1621 whose entries satisfy $a>b>-d \geq 0$. Some experimentation suggests

$$
A=\left(\begin{array}{cc}
1640 & 221 \\
-141 & -19
\end{array}\right)
$$

as an example of a matrix that fails the test for a monodromy of a CI: the cycle of $A$ is $(8,2,4,3,12)$, and the cycle of its dual has length 19 .

\section{Classifying Galois covers}

We now verify that the above choice for $A$ does indeed classify a cusp with no CI Galois cover. As mentioned in Section 2, we only need to consider covers of degrees $1,2,3$, and 4 in the base. In the notation of (2), the maximal indices in $\mathbb{Z}^{2}$ for the fiber subgroups of normal subgroups are $q, 3 r q, q(2 s)^{2}$, and $3 p^{2} q r$, respectively. With our choice of $A$ we have $p=1621, q=1619, r=541$, and $s=811$.

For each degree, we find a basis representation for the subgroups lying between $\mathbb{Z}^{2}$ and $\left(A^{n}-I\right) \mathbb{Z}^{2}$; by Proposition $[2.2$ the normal subgroups are those preserved by $A$. Once we have found such a subgroup, we need to know how a generator $g$ of $\mathbb{Z}$ acts on it; the matrix of this action (raised to the $n$th power, for degree $n$ ) will 
be the monodromy of the Galois cover. We can then use the machinery described in Section 3 to compute the classifying cycle of the cover; for computation, the algorithms were implemented in Mathematica. The action of $g$ on the maximalindex subgroup $\left(A^{n}-I\right) \mathbb{Z}^{2}$ is always by $A$, since $A$ commutes with $\left(A^{n}-I\right)$. So we only need to check subgroups properly intermediate between $\mathbb{Z}^{2}$ and $\left(A^{n}-I\right) \mathbb{Z}^{2}$.

In fact, we need to treat only half of the possible subgroups for each degree. The other half represent dual cusps, and we know how to compute their cycles from the original cusps. In general, if $\left[\mathbb{Z}^{2}:\left(A^{n}-I\right) \mathbb{Z}^{2}\right]=x y$, and $L$ is a subgroup of index $x$, then a subgroup $L^{\prime}$ of index $y$ represents the fiber of the dual cusp. (This is implied in Section 4 of [7.)

4.1. One-fold covers. This case is easy: If $N=L \rtimes_{B} \mathbb{Z}$, then $L=\mathbb{Z}^{2}$ or $L=$ $(A-I) \mathbb{Z}^{2}$. Since $\left[\mathbb{Z}^{2}:(A-I) \mathbb{Z}^{2}\right]=q$ is prime, there can be no intermediate subgroups.

4.2. Two-fold covers. This case is also fairly simple: $\left[\mathbb{Z}^{2}:\left(A^{2}-I\right) \mathbb{Z}^{2}\right]=3 r q$. So the intermediate subgroups are of index $3, q, 3 q, r q, 3 r$, and $r$. We will treat the first three, and consider the rest as dual to these.

Since the index of each subgroup in this case is a prime or a product of distinct primes, each subgroup is uniquely determined by its index. From the one-fold case, we already know that the index $q$ subgroup is $(A-I) \mathbb{Z}^{2}$ and does not change the action of $g$. The dual subgroup is then $(A+I) \mathbb{Z}^{2}$ and also does nothing to $g$.

Consider the index 3 subgroup as the kernel of a map $\mathbb{Z}^{2} \rightarrow \mathbb{Z}_{3}$. There are four distinct such mappings; their kernels are

$$
\begin{array}{l|l}
\left\{(x, y) \in \mathbb{Z}^{2}\right. & y \equiv 0 \quad(\bmod 3)\} \\
\left\{(x, y) \in \mathbb{Z}^{2}\right. & \mid \quad x \equiv 0 \quad(\bmod 3)\} \\
\left\{(x, y) \in \mathbb{Z}^{2}\right. & \mid x+y \equiv 0 \quad(\bmod 3)\}, \text { and } \\
\left\{(x, y) \in \mathbb{Z}^{2}\right. & \mid x-y \equiv 0 \quad(\bmod 3)\} .
\end{array}
$$

Modulo 3, our matrix $A$ is $\left(\begin{array}{cc}2 & 2 \\ 0 & 2\end{array}\right)$, and it is easy to check that only the first subgroup in (8) is preserved by $A$. The index 3 normal subgroup, then, is given by

$$
\mathbf{3}=\left\langle\left(\begin{array}{l}
1 \\
0
\end{array}\right),\left(\begin{array}{l}
0 \\
3
\end{array}\right)\right\rangle .
$$

Conjugating $A$ by $\left(\begin{array}{ll}1 & 0 \\ 0 & 3\end{array}\right)$ gives the action of $g$ on this subgroup; it is

$$
A_{3}=\left(\begin{array}{cc}
1640 & 663 \\
-47 & -19
\end{array}\right)
$$

Knowing the subgroups of index 3 and $q$, one can find the subgroup of index $3 q$ by acting on the index 3 subgroup with $(A-I)$. The action of $g$ on this subgroup is then given by $\left[(A-I)\left(\begin{array}{ll}1 & 0 \\ 0 & 3\end{array}\right)\right]^{-1} A\left[(A-I)\left(\begin{array}{ll}1 & 0 \\ 0 & 3\end{array}\right)\right]$, which is just $A_{3}$.

Calculating the cycle and dual cycle of $A_{3}$, we find they have lengths 4 and 42 . Therefore, the monodromy $\left(A_{\mathbf{3}}\right)^{2}$ has cycle and dual cycle of lengths 8 and 84 , well over the limit for complete intersections.

The index $r$ subgroup represents the cover dual to the one represented by the $3 q$ subgroup, but later we will need a basis representation for it in calculating four-fold covers. As in the index 3 case, the possible subgroups are generated by $\{(1,0),(0, r)\}$ or $\{(t, 1),(r, 0)\}$, for $0 \leq t<r$. Only the second of these is preserved 
by $A$. Conjugating by $\left(\begin{array}{ll}t & r \\ 1 & 0\end{array}\right)$ yields

$$
\left(\begin{array}{cc}
-19-141 t & -141 r \\
\frac{1}{r}\left(221+1659 t+141 t^{2}\right) & 1640+141 t
\end{array}\right),
$$

which suggests we solve

$$
221+1659 t+141 t^{2} \equiv 0 \quad(\bmod r) .
$$

For $r=541$, we find $t=138$ is the unique solution (modulo $r$ ). So the index $r$ subgroup is

$$
\mathbf{r}=\left\langle\left(\begin{array}{c}
138 \\
1
\end{array}\right),\left(\begin{array}{c}
541 \\
0
\end{array}\right)\right\rangle .
$$

4.3. Three-fold covers. Here we have $\left[\mathbb{Z}^{2}:\left(A^{3}-I\right) \mathbb{Z}^{2}\right]=q(2 s)^{2}$. So the group structure is somewhat more complicated: we have to contend with subgroups of index $s^{2}$, as well as subgroups of composite index. Here we will look at the subgroups of index $q, 2, s, 2 s, 2 q, s q, s^{2}$, and $s^{2} q$, the rest being dual to these. The index $q$ subgroup is still $(A-I) \mathbb{Z}^{2}$, and we can use the same strategy employed in the two-fold case to move from index $x$ to index $x q$, i.e., multiply by $(A-I)$. Thus only the index $2, s$, and $s^{2}$ cases require explicit calculation.

There are three mappings onto $\mathbb{Z}_{2}$, with kernels

$$
\begin{array}{l|l}
\left\{(x, y) \in \mathbb{Z}^{2}\right. & y \equiv 0 \quad(\bmod 2)\}, \\
\left\{(x, y) \in \mathbb{Z}^{2}\right. & x \equiv 0 \quad(\bmod 2)\}, \text { and } \\
\left\{(x, y) \in \mathbb{Z}^{2}\right. & x+y \equiv 0 \quad(\bmod 2)\} .
\end{array}
$$

But $A \equiv\left(\begin{array}{ll}0 & 1 \\ 1 & 1\end{array}\right) \quad(\bmod 2)$. So none of these subgroups is preserved. Therefore there is no normal cover of index 2 in the fiber.

Using the fact that there are no index 2 normal subgroups, a simple argument eliminates the $2 s$ and $2 q$ cases as well: Suppose one of these latter is normal; let $K$ be this normal fiberwise subgroup of $G$. But then $|G / K|=2 s$ or $2 q$, and in either case $G / K$ has a characteristic subgroup of order 2 . So there must be a normal index 2 subgroup of $G$. We already know this is not the case.

There are $s+1$ possibilities for index $s$ subgroups:

$$
\begin{array}{l|l}
\left\{(x, y) \in \mathbb{Z}^{2}\right. & y \equiv 0 \quad(\bmod s)\}, \text { and } \\
\left\{(x, y) \in \mathbb{Z}^{2}\right. & \mid x-t y \equiv 0 \quad(\bmod s), 0 \leq t<s\} .
\end{array}
$$

The requirement that $A$ preserve these subgroups is equivalent to asking that $P^{-1} A P \in S L_{2}(\mathbb{Z})$, where $P$ is a change of basis matrix. We can easily check that

$$
\left(\begin{array}{ll}
1 & 0 \\
0 & s
\end{array}\right)^{-1}\left(\begin{array}{cc}
1640 & 221 \\
-141 & -19
\end{array}\right)\left(\begin{array}{ll}
1 & 0 \\
0 & s
\end{array}\right)
$$

does not have integer entries. So the first subgroup is ruled out. For the other $s$ possibilities, conjugation by $\left(\begin{array}{ll}t & r \\ 1 & 0\end{array}\right)$ gives

$$
\left(\begin{array}{cc}
-19-141 t & -141 s \\
\frac{1}{s}\left(221+1659 t+141 t^{2}\right) & 1640+141 t
\end{array}\right) ;
$$

so as in (9)), we solve $221+1659 t+141 t^{2} \equiv 0(\bmod s)$. With $s=811$, we find $t=183$ and $t=668$ are the two solutions.

The two index $s$ normal subgroups are then

$$
\mathbf{s}_{\mathbf{1}}=\left\langle\left(\begin{array}{c}
183 \\
1
\end{array}\right),\left(\begin{array}{c}
811 \\
0
\end{array}\right)\right\rangle ; \quad \mathbf{s}_{\mathbf{2}}=\left\langle\left(\begin{array}{c}
668 \\
1
\end{array}\right),\left(\begin{array}{c}
811 \\
0
\end{array}\right)\right\rangle,
$$


and the action of $g$ on each is

$$
\begin{aligned}
& A_{\mathbf{s}_{1}}=\left(\begin{array}{cc}
-25822 & -114351 \\
6197 & 27443
\end{array}\right) ; \\
& A_{\mathbf{s}_{2}}=\left(\begin{array}{cc}
-94207 & -114351 \\
78947 & 95828
\end{array}\right) .
\end{aligned}
$$

We now calculate the relevant cycles, and find that the cycle and dual cycle of $\left(A_{\mathbf{s}_{\mathbf{1}}}\right)^{3}$ have lengths $3 \cdot 149$ and $3 \cdot 3$, respectively, while the cycle and dual of $\left(A_{\mathbf{s}_{\mathbf{2}}}\right)^{3}$ have lengths $3 \cdot 14$ and $3 \cdot 12$. None of these, then, is a complete intersection.

From the Sylow theorems we know that there is exactly one order $s^{2}$ subgroup of $\mathbb{Z}^{2} /\left(A^{3}-I\right) \mathbb{Z}^{2}$. So there can be only one normal subgroup of $\mathbb{Z}^{2}$ of index $s^{2}$. We can easily check that this subgroup is

$$
\mathbf{s}^{2}=\left\langle\left(\begin{array}{l}
s \\
0
\end{array}\right),\left(\begin{array}{l}
0 \\
s
\end{array}\right)\right\rangle \text {. }
$$

Since $s$ divides $\left(A^{3}-I\right)=(A-I)\left(A^{2}+A+I\right),\left(A^{3}-I\right) \mathbb{Z}^{2} \subset\left(\begin{array}{ll}s & 0 \\ 0 & s\end{array}\right) \mathbb{Z}^{2}$. Also, this subgroup is clearly preserved by $A$, since

$$
A_{\mathbf{s}^{2}}=\left(\begin{array}{ll}
s & 0 \\
0 & s
\end{array}\right)^{-1} A\left(\begin{array}{ll}
s & 0 \\
0 & s
\end{array}\right)=A .
$$

We are now left with the $s q$ and $s^{2} q$ cases. These subgroups are found simply by acting with $(A-I)$ on the bases of the index $s$ and index $s^{2}$ subgroups. If $P$ represents the change of basis from one of these to $\mathbb{Z}^{2}$, then $(A-I) P$ is the change of basis for the corresponding subgroup with the added factor of $q$. Then $[(A-I) P]^{-1} A[(A-I) P]=P^{-1}(A-I)^{-1} A(A-I) P=P^{-1} A P$. So we have

$$
\begin{aligned}
& A_{(\mathbf{s q})_{i}}=A_{\mathbf{s}_{i}}(i=1,2), \\
& A_{\left(\mathbf{s}^{2} \mathbf{q}\right)}=A_{\mathbf{s}^{2}},
\end{aligned}
$$

and we have already examined both of these matrices.

4.4. Four-fold covers. This case is similar to the three-fold case, but simplified by the fact that we have already treated most of the relevant subgroups. The index is now $3 p^{2} q r$, and we can use the same duality argument to focus our attention on half of the subgroups; of these, only those of index $p, q p, r p$, and $3 p$ have not been discussed above.

As in the three-fold case, the $p+1$ subgroups of index $p$ are

$$
\begin{array}{l|l}
\left\{(x, y) \in \mathbb{Z}^{2}\right. & y \equiv 0 \quad(\bmod p)\} \text { and } \\
\left\{(x, y) \in \mathbb{Z}^{2}\right. & \mid x-t y \equiv 0 \quad(\bmod p), 0 \leq t<p\}
\end{array}
$$

Again, the first of these can be immediately eliminated, since it is not preserved by $A$. We then solve as before for $t$ such that $221+1659 t+141 t^{2} \equiv 0(\bmod p)$, finding $t=139$ or $t=608$.

Thus, the two index $p$ normal covers are

$$
\mathbf{p}_{\mathbf{1}}=\left\langle\left(\begin{array}{c}
139 \\
1
\end{array}\right),\left(\begin{array}{c}
1621 \\
0
\end{array}\right)\right\rangle ; \quad \mathbf{p}_{\mathbf{2}}=\left\langle\left(\begin{array}{c}
608 \\
1
\end{array}\right),\left(\begin{array}{c}
1621 \\
0
\end{array}\right)\right\rangle
$$

and the action of $g$ on each is

$$
\begin{aligned}
& A_{\mathbf{p}_{1}}=\left(\begin{array}{cc}
-19618 & -228561 \\
1823 & 21239
\end{array}\right), \\
& A_{\mathbf{p}_{2}}=\left(\begin{array}{cc}
-85747 & -228561 \\
32777 & 87368
\end{array}\right) .
\end{aligned}
$$


The cycle and dual cycle of $\left(A_{\mathbf{p}_{1}}\right)^{4}$ have lengths $4 \cdot 12$ and $4 \cdot 10$, respectively; the cycle and dual of $\left(A_{\mathbf{p}_{\mathbf{2}}}\right)^{4}$ have lengths $4 \cdot 101$ and $4 \cdot 3$. None of these is a CI.

We can find the composite-index subgroups using a method similar to the one used in the three-fold case. As in that case, the action does not change as we move from the index $p$ to the index $q p$ subgroups:

$$
A_{(\mathbf{q p})_{i}}=A_{\mathbf{p}_{i}} .
$$

Using the representation of the index $r$ subgroup found in Section 4.2, we can calculate the normal index $r p$ subgroups. Now we want

$$
P_{t}^{-1} A P_{t} \in S L_{2}(\mathbb{Z}),
$$

where $P_{t}=\left(\begin{array}{cc}138 & 541 \\ 1 & 0\end{array}\right)\left(\begin{array}{ll}t & p \\ 1 & 0\end{array}\right)$. This reduces to solving

$$
1527+1571 t+1097 t^{2} \equiv 0 \quad(\bmod p) .
$$

We find that $t=541$ and $t=653$ solve this, and so the actions of $g$ on the two index $r p$ subgroups are

$$
\begin{aligned}
& A_{(\mathbf{r p})_{1}}=P_{541}^{-1} A P_{541}=\left(\begin{array}{cc}
2935465 & 8732327 \\
-986243 & -2933844
\end{array}\right), \\
& A_{(\mathbf{r p})_{2}}=P_{653}^{-1} A P_{653}=\left(\begin{array}{cc}
3538809 & 8732327 \\
-1433459 & -3537188
\end{array}\right),
\end{aligned}
$$

where

$$
P_{541}=\left(\begin{array}{cc}
138 & 541 \\
1 & 0
\end{array}\right)\left(\begin{array}{cc}
541 & 1621 \\
1 & 0
\end{array}\right)
$$

and

$$
P_{653}=\left(\begin{array}{cc}
138 & 541 \\
1 & 0
\end{array}\right)\left(\begin{array}{cc}
653 & 1621 \\
1 & 0
\end{array}\right) \text {. }
$$

The classifying matrix $\left(A_{\left(\mathbf{r p}_{\mathbf{1}}\right)}\right)^{4}$ has cycle length $4 \cdot 8$ and dual cycle length $4 \cdot 10$, and $\left(A_{(\mathbf{r p})_{2}}\right)^{4}$ has cycle length $4 \cdot 8$ and dual cycle length $4 \cdot 34$; the index $r p$ covers, then, are not complete intersections.

Finally, we turn to the index $3 p$ subgroups. In fact, we have already calculated the monodromies and cycles for these. Recall that adding a factor of $q$ to the index leaves the monodromy unchanged; thus the index $3 p$ covers have the same monodromies as the index $3 p q$ covers. But the index $3 p q$ covers are dual to the index $r p$ covers, whose classifying matrices (and duals) were computed above. This completes our verification that the cusp classified by $A$, with cycle $(8,2,4,3,12)$, has no Galois cover by a complete intersection.

\section{REFERENCES}

[1] F. Hirzebruch, Hilbert modular surfaces, Enseignement Math. 19 (1973), no. 2, 183-281. MR 52:13856

[2] U. Karras, Eigenschaften der lokalen Ringe in zweidimensionalen Spitzen, Math. Ann. 215 (1975), 117-129. MR 51:10679

[3] Ramanujachary Kumanduri and Cristina Romero, Number theory with computer applications, ch. 11, pp. 270-271, Prentice Hall, 1998.

[4] W. D. Neumann, A calculus for plumbing applied to the topology of complex surface singularities and degenerating complex curves, Trans. Amer. Math. Soc. 268 (1981), no. 2, 299-344. MR 84a:32015

[5] W. D. Neumann, Abelian covers of quasihomogeneous surface singularities, Singularities, Arcata 1981, Proc. Sympos. Pure Math., vol. 40, Amer. Math. Soc., Providence, RI, 1983, pp. 233-243. MR 85g:32018 
[6] W. D. Neumann and Jonathan Wahl, Casson invariant of links of singularities, Comment. Math. Helv. 65 (1990), 58-78. MR 91c:57022

[7] W. D. Neumann and Jonathan Wahl, Universal abelian covers of quotient-cusps, Math. Ann. 326 (2003), 75-93. MR 2004d:32039

Department of Mathematics, Columbia University, New York, New York 10027

E-mail address: anderson@math.columbia.edu

Current address: Department of Mathematics, University of Michigan, 2074 East Hall, Ann Arbor, Michigan 48109

E-mail address: dandersn@umich.edu 